\title{
ON THE CONSERVATION OF CROSS HELICITY AND WAVE ACTION IN SOLAR-WIND MODELS WITH NON-WKB ALFVÉN WAVE REFLECTION
}

\author{
Benjamin D. G. Chandran, Jean C. Perez, Daniel Verscharen, Kristopher G. Klein, and Alfred Mallet \\ Space Science Center and Department of Physics, University of New Hampshire, Durham, NH 03824, USA; benjamin.chandran@unh.edu \\ Received 2015 May 26; accepted 2015 August 13; published 2015 September 18
}

\begin{abstract}
The interaction between Alfvén-wave turbulence and the background solar wind affects the cross helicity $\left(\int d^{3} x \boldsymbol{v} \cdot \boldsymbol{B}\right)$ in two ways. Non-WKB reflection converts outward-propagating Alfvén waves into inwardpropagating Alfvén waves and vice versa, and the turbulence transfers momentum to the background flow. When both effects are accounted for, the total cross helicity is conserved. In the special case that the background density and flow speed are independent of time, the equations of cross-helicity conservation and total-energy conservation can be combined to recover a well-known equation derived by Heinemann and Olbert that has been interpreted as a non-WKB generalization of wave-action conservation. This latter equation (in contrast to cross-helicity and energy conservation) does not hold when the background varies in time.
\end{abstract}

Key words: magnetohydrodynamics (MHD) - solar wind - Sun: corona - turbulence - waves

\section{INTRODUCTION}

Approximately fifty years ago, Parker (1965) and Coleman (1968) suggested that waves and turbulence play an important role in the heating and acceleration of the solar wind. Since that time, observational, theoretical, and numerical studies have produced mounting evidence that supports this suggestion. For example, in situ measurements at heliocentric distances exceeding $0.3 \mathrm{AU}$ show that turbulent fluctuations pervade the interplanetary medium (Goldstein et al. 1995; Bruno \& Carbone 2005) and that most of this turbulence consists of fluctuations that propagate away from the Sun, consistent with a solar origin (Belcher \& Davis 1971; Tu \& Marsch 1995). Remote observations from the Solar Optical Telescope on the Hinode satellite reveal the presence of Alfvén-wave-like motions in the low corona with amplitudes sufficient to power the solar wind (De Pontieu et al. 2007). Faraday rotation of radio transmissions from the Helios satellite are also consistent with theoretical models in which the solar wind is powered by an Alfvén-wave (AW) energy flux (Hollweg et al. 2010).

In order for AW turbulence to heat the solar wind, AW energy that is initially in large-wavelength fluctuations must "cascade" to smaller wavelengths, at which the fluctuations can efficiently dissipate. This cascade process relies upon the interaction between counter-propagating AWs (Iroshnikov 1963; Kraichnan 1965). Because the Sun launches only outward-propagating waves, solar-wind heating by AW turbulence requires some source of inward-propagating AWs.

One of the most important sources of such inwardpropagating waves is non-WKB reflection (Heinemann \& Olbert 1980; Velli 1993; Hollweg \& Isenberg 2007). Photospheric motions have such long timescales that they launch AWs that have radial wavelengths within the corona and solar wind that can be comparable to or greater than the heliocentric distance. For such waves, the wave phase velocity varies appreciably over one wave length, which causes the AWs to undergo partial reflection as they propagate away from the Sun.

A number of authors have conducted theoretical and numerical investigations of solar-wind turbulence driven by non-WKB AW reflection (e.g., Zhou \& Matthaeus 1989; Velli et al. 1989; Matthaeus et al. 1999; Dmitruk et al. 2002;
Cranmer \& van Ballegooijen 2005; Verdini \& Velli 2007; Chandran \& Hollweg 2009; Verdini et al. 2012; Perez \& Chandran 2013). These authors took the background solar wind to be steady, and several of them made use of a conservation law first obtained by Heinemann \& Olbert (1980), which Heinemann \& Olbert (1980) interpreted as a non-WKB generalization of wave-action conservation. In this paper, we show that this conservation relation does not hold in the case of a time-dependent background. We also show that this conservation relation can be obtained by combining the equations of cross-helicity conservation and energy conservation. To the best of our knowledge, the equation of crosshelicity conservation has not been applied previously to reflection-driven AW turbulence in the solar wind. Because the equation of "non-WKB wave-action conservation" can be obtained from the equation of cross-helicity conservation, and because cross helicity is conserved regardless of whether the background varies in time, the equation of cross-helicity conservation in some sense generalizes the equation of "nonWKB wave-action conservation" to the time-dependent regime. We note that although we allow the flow velocity and density to vary in time, our analysis is limited to the case in which the background magnetic field is fixed.

In Section 2, we describe and adopt a standard set of approximations that has been used in previous treatments of non-WKB reflection of Alfvén waves in the solar wind. For example, we assume that the background magnetic field is quasi-radial (Section 2.1) and that the fluctuations are transverse and non-compressive (Section 2.3). We then summarize the derivation of the equations that describe the coupled evolution of the fluctuations and the background flow (Section 2.4). In Section 3, we describe how the equations in Section 2.4 lead to conservation equations for the total energy and total cross helicity. We also show how these two conservation equations can be combined to recover the aforementioned equation of "non-WKB wave-action conservation" when the background flow is independent of time. Finally, in Section 4, we describe how the equations in our model separately conserve the action of inward and outwardpropagating AWs in the limit of short wavelengths and small wave amplitudes. 


\section{COUPLED EQUATIONS FOR THE FLUCTUATING FIELDS AND BACKGROUND FLOW}

We begin with the equations of ideal magnetohydrodynamics (MHD),

$$
\begin{aligned}
\frac{\partial \rho}{\partial t} & =-\nabla \cdot(\rho \boldsymbol{v}) \\
\rho\left(\frac{\partial \boldsymbol{v}}{\partial t}+\boldsymbol{v} \cdot \nabla \boldsymbol{v}\right)= & -\nabla\left(p+\frac{B^{2}}{8 \pi}\right)+\frac{\boldsymbol{B} \cdot \nabla \boldsymbol{B}}{4 \pi} \\
& -\frac{G M_{\odot} \rho \hat{\boldsymbol{r}}}{r^{2}}
\end{aligned}
$$

and

$$
\frac{\partial \boldsymbol{B}}{\partial t}=\nabla \times(\boldsymbol{v} \times \boldsymbol{B}),
$$

where $\rho$ is the mass density, $\boldsymbol{v}$ is the velocity, $\boldsymbol{B}$ is the magnetic field, $p$ is the pressure, $G$ is the gravitational constant, and $M_{\odot}$ is the mass of the Sun. We assume that the plasma satisfies the energy equation

$$
\frac{1}{\gamma-1}\left[\frac{\partial p}{\partial t}+\nabla \cdot(\boldsymbol{v} p)\right]=-p \nabla \cdot \boldsymbol{v}-\nabla \cdot \boldsymbol{q},
$$

where $\boldsymbol{q}$ is the heat flux and $\gamma$ is the ratio of specific heats.

\subsection{Two Models for the Background Magnetic Field}

We set

$$
\boldsymbol{B}=\boldsymbol{B}_{0}+\delta \boldsymbol{B},
$$

where the background magnetic field $\boldsymbol{B}_{0}$ is a fixed, timeindependent function of space. We neglect solar rotation and consider two models for the background magnetic field:

Model 1: $\boldsymbol{B}_{0}$ consists of exactly radial magnetic field lines filling a region that spans a solid angle of order unity as seen from the Sun, with $B_{0}$ depending only on heliocentric distance $r$.

Model 2: $\boldsymbol{B}_{0}$ corresponds to a narrow magnetic flux tube in which the magnetic field is nearly but not exactly radial, which allows for super-radial divergence of the magnetic field. In this second case, we take the flux-tube to have an approximately square cross section and impose periodic boundary conditions on the edges of this cross section, as described further in Appendix A. We also take the opening angle $\theta$ of the flux tube to be $\ll 1$.

In both models,

$$
\boldsymbol{a} \cdot \nabla \hat{\boldsymbol{b}}=\frac{\sigma}{2} \boldsymbol{a}_{\perp}
$$

for any vector $\boldsymbol{a}$, where

$$
\begin{gathered}
\boldsymbol{a}_{\perp} \equiv \boldsymbol{a}-\hat{\boldsymbol{b}}(\boldsymbol{a} \cdot \hat{\boldsymbol{b}}), \\
\sigma=\nabla \cdot \hat{\boldsymbol{b}},
\end{gathered}
$$

and

$$
\hat{\boldsymbol{b}}=\frac{\boldsymbol{B}_{0}}{B_{0}} .
$$

Equation (6) is exact in model 1 and correct to leading order in $\theta$ in model 2 . In both models, we are able to discard terms proportional to either $\nabla \times \hat{\boldsymbol{b}}$ or $\hat{\boldsymbol{b}} \cdot \nabla \hat{\boldsymbol{b}}$. Such terms vanish exactly in the case of model 1 and are much smaller than the terms we keep in the case of model 2.

\subsection{Averages over Surfaces Perpendicular to $\boldsymbol{B}_{0}$}

For both background-magnetic-field models discussed in Section 2.1, we define the "surface average" of an arbitrary function $f$, denoted $\langle f\rangle$, through the equation

$$
\langle f\rangle=\frac{1}{A} \int_{S} d A f
$$

where $\int_{S} d A f$ denotes an integral of $f$ over a surface $S$ that has area $A$ and is everywhere normal to $\boldsymbol{B}_{0}$. In model $1, S$ is the intersection of the modeled region with a spherical shell of some radius $r$. In model 2, the surface integral in Equation (10) is confined to the interior of the modeled magnetic flux tube. In both models, we are able to discard terms of the form $\left\langle\nabla \cdot \boldsymbol{a}_{\perp}\right\rangle$ when they arise in the derivation of the equations in Section 2.4, where $\boldsymbol{a}_{\perp}$ is everywhere perpendicular to $\hat{\boldsymbol{b}}$, because such terms are much smaller than the terms we keep. We discuss this point further in the context of model 2 in Appendix A. In model 1, averages of the form $\left\langle\nabla \cdot \boldsymbol{a}_{\perp}\right\rangle$ can be dropped when $\boldsymbol{a}_{\perp}$ contains one or more fluctuating quantities in part because we assume that

$$
L_{\mathrm{c} \perp} \ll r,
$$

where $L_{\mathrm{c} \perp}$ is the correlation length of the turbulence perpendicular to $\boldsymbol{B}_{0}$.

\subsection{The Transverse, Non-Compressive Approximation}

As in Equation (5), we set each of $\rho, v$, and $p$ equal to the sum of a background value (denoted with a " 0 " subscript) and a fluctuating part $(\delta \rho, \delta v$, and $\delta p)$. We define

$$
\boldsymbol{v}=v_{\|} \hat{b}+\delta v_{\perp}
$$

where $\delta \boldsymbol{v}_{\perp} \cdot \hat{\boldsymbol{b}}=0$. For the scalar quantities $v_{\|}, p$, and $\rho$, we define the background quantities to be surface averages:

$$
U \equiv v_{\| 0}=\left\langle v_{\|}\right\rangle \quad p_{0}=\langle p\rangle \quad \rho_{0}=\langle\rho\rangle .
$$

We assume that

$$
\begin{gathered}
\nabla \cdot \boldsymbol{\delta} \boldsymbol{v}_{\perp}=0, \\
\boldsymbol{\delta} \boldsymbol{B} \cdot \hat{\boldsymbol{b}}=0, \\
\delta v_{\|} \equiv v_{\|}-U=0,
\end{gathered}
$$

and

$$
\delta \rho \ll \rho_{0} \quad \delta p \ll p_{0} .
$$

We refer to Equations (14) through (17) as the transverse, noncompressive approximation. Observations provide some support for this approximation. For example, in situ measurements show that turbulent fluctuations in the solar wind are weakly compressive and preferentially transverse (see, e.g., Goldstein et al. 1995; Tu \& Marsch 1995; Bruno \& Carbone 2005). Radio-scintillation observations further indicate that $\delta \rho \ll \rho_{0}$ at heliocentric distances as small as a few solar radii (Coles \& Harmon 1989; Markovskii \& Hollweg 2002; Chandran et al. 2009). On the other hand, transverse, non-compressive fluctuations nonlinearly generate compressive fluctuations and 
longitudinal fluctuations at some level, an effect that we neglect. Thus, while the transverse, non-compressive approximation may apply to the bulk of the fluctuation energy, the equations we derive in Section 2.4 do not account for all of the physical processes occurring in solar-wind turbulence.

\subsection{Coupled Equations for the Fluctuating and Background Quantities}

In this section, we present the equations that describe the fluctuations and background flow to leading order in $\theta, L_{\mathrm{c} \perp} / r$, $\delta \rho / \rho_{0}$, and $\delta p / p_{0}$. To obtain an equation describing the average parallel velocity, we take the dot product of Equation (2) with $\hat{\boldsymbol{b}}$ and then average the resulting equation over a surface perpendicular to $\hat{\boldsymbol{b}}$ as described in Section 2.2. This yields

$$
\begin{gathered}
\rho_{0}\left(\frac{\partial U}{\partial t}+U \frac{\partial U}{\partial r}\right)=-\frac{\partial}{\partial r}\left(p_{0}+\frac{\left\langle|\delta \boldsymbol{B}|^{2}\right\rangle}{8 \pi}\right) \\
+\frac{\sigma}{2}\left(\rho_{0}\left\langle\left|\boldsymbol{\delta} \boldsymbol{v}_{\perp}\right|^{2}\right\rangle-\frac{\left\langle|\boldsymbol{\delta} \boldsymbol{B}|^{2}\right\rangle}{4 \pi}\right)-\frac{G M_{\odot} \rho_{0}}{r^{2}} .
\end{gathered}
$$

The term proportional to $\sigma$ on the right-hand side of Equation (18) is the radial component of the averaged MHD Reynolds stress $\left\langle\boldsymbol{\delta} \boldsymbol{B} \cdot \nabla \boldsymbol{\delta} \boldsymbol{B} / 4 \pi-\rho_{0} \boldsymbol{\delta} \boldsymbol{v}_{\perp} \cdot \nabla \delta \boldsymbol{v}_{\perp}\right\rangle$, and was obtained previously by Usmanov et al. (2011, 2014).

Upon multiplying Equation (18) by $\hat{\boldsymbol{b}}$ and subtracting the resulting equation from Equation (2), we obtain the perpendicular momentum equation,

$$
\begin{aligned}
\rho_{0} \frac{\partial}{\partial t} \boldsymbol{\delta} \boldsymbol{v}_{\perp} & +\rho_{0} \boldsymbol{U} \cdot \nabla \boldsymbol{\delta} \boldsymbol{v}_{\perp}+\frac{\rho_{0} \sigma U}{2} \boldsymbol{\delta} \boldsymbol{v}_{\perp}=-\nabla_{\perp}(\delta \Pi) \\
& +\frac{\boldsymbol{B}_{0} \cdot \nabla \boldsymbol{\delta} \boldsymbol{B}}{4 \pi}+\frac{\sigma B_{0} \boldsymbol{\delta} \boldsymbol{B}}{8 \pi} \\
& +\left(\frac{\boldsymbol{\delta} \cdot \nabla \boldsymbol{\delta} \boldsymbol{B}}{4 \pi}-\rho_{0} \boldsymbol{\delta} \boldsymbol{v}_{\perp} \cdot \nabla \delta \boldsymbol{v}_{\perp}\right)_{\perp}
\end{aligned}
$$

where

$$
\boldsymbol{U}=U \hat{\boldsymbol{b}}
$$

$\delta \Pi$ is the fluctuating part of $p+B^{2} / 8 \pi$, the quantity $(\boldsymbol{a})_{\perp}$ is defined via Equation (7) for arbitrary $\boldsymbol{a}$, and $\nabla_{\perp} f=(\nabla f)_{\perp}$ for an arbitrary scalar function $f$. The projection of Equation (3) onto a plane perpendicular to $\hat{\boldsymbol{b}}$ yields

$$
\begin{gathered}
\frac{\partial}{\partial t} \boldsymbol{\delta} \boldsymbol{B}=\boldsymbol{B}_{0} \cdot \nabla \boldsymbol{\delta} \boldsymbol{v}_{\perp}-\boldsymbol{U} \cdot \nabla \boldsymbol{\delta} \boldsymbol{B}+\frac{\sigma}{2}\left(U \boldsymbol{\delta} \boldsymbol{B}-B_{0} \boldsymbol{\delta} \boldsymbol{v}_{\perp}\right) \\
-\boldsymbol{\delta} \boldsymbol{B} \nabla \cdot \boldsymbol{U}+\left(\boldsymbol{\delta} \boldsymbol{B} \cdot \nabla \boldsymbol{\delta} \boldsymbol{v}_{\perp}-\boldsymbol{\delta} \boldsymbol{v}_{\perp} \cdot \nabla \boldsymbol{\delta} \boldsymbol{B}\right)_{\perp}
\end{gathered}
$$

Averaging Equation (1) as in Section 2.2, we obtain

$$
\frac{\partial \rho_{0}}{\partial t}=-\nabla \cdot\left(\rho_{0} \boldsymbol{U} \hat{\boldsymbol{b}}\right) .
$$

Subtracting Equation (22) from (1), we find that

$$
\frac{\partial}{\partial t} \delta \rho+\delta \boldsymbol{v}_{\perp} \cdot \nabla \delta \rho=-\nabla \cdot(\delta \rho \boldsymbol{U} \hat{\boldsymbol{b}}) .
$$

Because of the transverse, non-compressive approximation (Equations (14) through (17)), the density fluctuations have no effect on the flow to leading order. Equation (23) thus describes the evolution of passive-scalar density fluctuations in the expanding solar wind.

We define a normalized magnetic fluctuation

$$
\boldsymbol{\delta} \boldsymbol{w}=\frac{\boldsymbol{\delta} \boldsymbol{B}}{\sqrt{4 \pi \rho_{0}}}
$$

and the Elsasser variables

$$
z^{ \pm}=\delta v_{\perp} \mp \delta w .
$$

Given our sign convention in Equation $(25), z^{+}\left(z^{-}\right)$represents non-compressive, Alfvén-wave-like fluctuations that propagate in the direction of $\boldsymbol{B}_{0}\left(-\boldsymbol{B}_{0}\right)$. By combining Equations (19) and (21), we find that

$$
\begin{aligned}
& \frac{\partial}{\partial t} \boldsymbol{z}^{ \pm}+\left(\boldsymbol{U} \pm \boldsymbol{v}_{\mathrm{A}}\right) \cdot \nabla \boldsymbol{z}^{ \pm}=-\nabla_{\perp}(\delta \Pi)-\frac{\sigma}{2}\left(U \mp v_{\mathrm{A}}\right) \boldsymbol{z}^{\mp} \\
& +\frac{\left(\boldsymbol{z}^{+}-\boldsymbol{z}^{-}\right)}{2}\left(\nabla \cdot \boldsymbol{v}_{\mathrm{A}} \mp \frac{1}{2} \nabla \cdot \boldsymbol{U}\right)-\left(\boldsymbol{z}^{\mp} \cdot \nabla \boldsymbol{z}^{ \pm}\right)_{\perp},
\end{aligned}
$$

where

$$
\boldsymbol{v}_{\mathrm{A}} \equiv \frac{\boldsymbol{B}_{0}}{\sqrt{4 \pi \rho_{0}}}
$$

is the Alfvén velocity. Equation (26) was previously used by Chandran \& Hollweg (2009) and is a specialized form of the more general Elsasser-variable equation obtained by a number of authors (e.g., Zhou \& Matthaeus 1990; Velli 1993; Verdini \& Velli 2007; Zank et al. 2012), in which we have used Equation (6) to replace the quantity $z^{ \pm} \cdot \nabla\left(-\boldsymbol{U} \pm \boldsymbol{v}_{\mathrm{A}}\right)$ appearing in those studies with the quantity $(\sigma / 2) z^{ \pm}\left(-U \pm v_{\mathrm{A}}\right)$. We rewrite Equation (26) in terms of the Elsasser stream functions and Elsasser vorticities in Appendix B.

\section{CONSERVATION LAWS}

To obtain an equation expressing conservation of total energy, we first take the dot product of Equation (26) with $2 z^{ \pm}$ and average over a surface perpendicular to $\hat{b}$ to find

$$
\begin{gathered}
\frac{\partial}{\partial t}\left\langle\left(z^{ \pm}\right)^{2}\right\rangle+\left(\boldsymbol{U} \pm \boldsymbol{v}_{\mathrm{A}}\right) \cdot \nabla\left\langle\left(z^{ \pm}\right)^{2}\right\rangle=-\sigma\left(U \mp v_{\mathrm{A}}\right)\left\langle z^{+} \cdot \boldsymbol{z}^{-}\right\rangle \\
\pm\left\langle\left(z^{ \pm}\right)^{2}-\boldsymbol{z}^{+} \cdot \boldsymbol{z}^{-}\right\rangle\left(\nabla \cdot \boldsymbol{v}_{\mathrm{A}} \mp \frac{1}{2} \nabla \cdot \boldsymbol{U}\right) .
\end{gathered}
$$

We then take the sum of the following equations: Equation (18) multiplied by $U$; Equation (22) multiplied by $U^{2} / 2$; the "plus version" of Equation (28) multiplied by $\rho_{0} / 4$; the "minus version" of Equation (28) multiplied by $\rho_{0} / 4$; and the average of Equation (4) over a surface perpendicular to $\hat{\boldsymbol{b}}$. This yields

$$
\frac{\partial \mathcal{E}_{\mathrm{tot}}}{\partial t}+\nabla \cdot \boldsymbol{F}_{\mathrm{tot}}=0
$$

where

$$
\mathcal{E}_{\text {tot }}=\frac{\rho_{0} U^{2}}{2}+\frac{p_{0}}{\gamma-1}+\rho_{0} \Phi+\mathcal{E}_{\text {fluct }}
$$


is the surface-averaged total-energy density,

$$
\mathcal{E}_{\text {fluct }}=\frac{\rho_{0}}{4}\left\langle\left(z^{+}\right)^{2}+\left(z^{-}\right)^{2}\right\rangle
$$

is the energy density of the turbulent fluctuations,

$$
\Phi=-\frac{G M_{\odot}}{r}
$$

is the gravitational potential, and

$$
\begin{gathered}
\boldsymbol{F}_{\text {tot }}=\boldsymbol{U}\left(\frac{\rho_{0} U^{2}}{2}+\frac{\gamma p_{0}}{\gamma-1}+\rho_{0} \Phi+\mathcal{E}_{\text {fluct }}+\frac{\left\langle(\delta B)^{2}\right\rangle}{8 \pi}\right) \\
+\frac{\rho_{0} \boldsymbol{v}_{\mathrm{A}}}{4}\left\langle\left(z^{+}\right)^{2}-\left(z^{-}\right)^{2}\right\rangle+\langle\boldsymbol{q}\rangle
\end{gathered}
$$

is the surface-averaged total-energy flux.

The surface-averaged cross-helicity density is

$$
\mathcal{H}_{\mathrm{c}}=\langle\boldsymbol{v} \cdot \boldsymbol{B}\rangle=U B_{0}+\frac{\sqrt{\pi \rho_{0}}}{2}\left\langle\left(z^{-}\right)^{2}-\left(z^{+}\right)^{2}\right\rangle .
$$

We obtain the equation expressing total-cross-helicity conservation by adding the following equations: the "minus version" of Equation (28) multiplied by $\sqrt{\pi \rho_{0}} / 2$; the "plus version" of Equation (28) multiplied by $-\sqrt{\pi \rho_{0}} / 2$; and Equation (18) multiplied by $B_{0} / \rho_{0}$. This yields

$$
\frac{\partial \mathcal{H}_{\mathrm{c}}}{\partial t}+\nabla \cdot \boldsymbol{F}_{\mathrm{c}}=0
$$

where

$$
\boldsymbol{F}_{\mathrm{c}}=\boldsymbol{B}_{0}\left(\frac{U^{2}}{2}-\frac{\left\langle\delta v_{\perp}^{2}\right\rangle}{2}+\Phi+h\right)+\boldsymbol{U}\left\langle\boldsymbol{\delta} \boldsymbol{v}_{\perp} \cdot \boldsymbol{\delta} \boldsymbol{B}\right\rangle
$$

is the surface-averaged cross-helicity flux, and

$$
h(r)=\int d r \frac{1}{\rho_{0}} \frac{d p_{0}}{d r} .
$$

The fact that cross helicity is conserved in the presence of non-WKB wave reflection is perhaps surprising. Most studies of incompressible MHD turbulence focus on the case of a stationary background, in which the cross helicity arises entirely from the turbulent fluctuations. In that case, converting $z^{ \pm}$fluctuations into $z^{\mp}$ fluctuations would violate cross-helicity conservation. In contrast, the interaction between fluctuations and a moving, inhomogeneous, and time-dependent solar wind changes the cross helicity via two mechanisms: non-WKB wave reflection, which changes the cross helicity in the fluctuations, and the transfer of momentum from the fluctuations to the background plasma, which alters the cross-helicity content of the background flow. The combined effect of these two mechanisms conserves the total cross helicity in the sense of Equation (35), which, when integrated over some arbitrary volume, implies that the change in the total cross helicity within that volume equals the amount of cross helicity that flows into that volume through its boundaries.

We note that total cross helicity is also conserved in weak, homogeneous, compressible MHD turbulence, despite the fact that interactions between Alfvén waves and magnetosonic waves convert $z^{ \pm}$energy into $z^{\mp}$ energy (Chandran 2008). In that problem, there is no flow of cross helicity through the boundaries, and the change in the cross helicity of the fluctuations is exactly offset by the change in the cross helicity of the background. The cross helicity of the background changes because the resonant three-wave interactions that convert $z^{ \pm}$fluctuations into $z^{\mp}$ fluctuations simultaneously generate a small, average, background flow parallel or antiparallel to $\boldsymbol{B}_{0}$.

We can combine the equations of cross-helicity conservation and energy conservation by first multiplying Equation (35) by $\rho_{0} U / B_{0}$ and then using Equation (29) to rewrite the term $\left(\rho_{0} U / B\right) \nabla \cdot\left(\boldsymbol{B}_{0} h\right)=\boldsymbol{U} \cdot \nabla p_{0}$ in terms of variables other than $p_{0}$. Recalling that $\boldsymbol{B}_{0}$ and $\boldsymbol{U}$ are parallel, which implies that $\boldsymbol{B}_{0} \cdot \nabla\left(\rho_{0} U / B_{0}\right)=-\partial \rho_{0} / \partial t$, we find after some algebra that

$$
\begin{aligned}
\frac{\partial}{\partial t}\left[\frac{\rho_{0}\left(U+v_{\mathrm{A}}\right)\left\langle\left(z^{+}\right)^{2}\right\rangle}{4 v_{\mathrm{A}}}-\frac{\rho_{0}\left(U-v_{\mathrm{A}}\right)\left\langle\left(z^{-}\right)^{2}\right\rangle}{4 v_{\mathrm{A}}}\right] \\
+\nabla \cdot\left[\frac{\hat{\boldsymbol{b}} \rho_{0}\left(U+v_{\mathrm{A}}\right)^{2}\left\langle\left(z^{+}\right)^{2}\right\rangle}{4 v_{\mathrm{A}}}-\frac{\hat{\boldsymbol{b}} \rho_{0}\left(U-v_{\mathrm{A}}\right)^{2}\left\langle\left(z^{-}\right)^{2}\right\rangle}{4 v_{\mathrm{A}}}\right] \\
=-\frac{\rho_{0}\left\langle\boldsymbol{\delta} \boldsymbol{v}_{\perp} \cdot \boldsymbol{\delta} \boldsymbol{B}\right\rangle}{B_{0}} \frac{\partial U}{\partial t}-\frac{\left\langle\left(\delta v_{\perp}\right)^{2}\right\rangle}{2} \frac{\partial \rho_{0}}{\partial t} .
\end{aligned}
$$

When the background plasma is steady, the right-hand side of Equation (38) vanishes, and Equation (38) is equivalent to Equation (26) of Heinemann \& Olbert (1980), which those authors interpreted as the generalization of AW action conservation to the non-WKB regime. Although Heinemann \& Olbert (1980) derived their Equation (26) for linear waves, their Equation (26) is also valid in the nonlinear regime, provided $\partial \rho_{0} / \partial t=\partial U / \partial t=0$, as can be seen from Equation (38) above. On the other hand, when the background plasma varies in time, the right-hand side of Equation (38) is in general nonzero. Thus, Heinemann \& Olbert (1980)'s Equation (26) does not extend to the case of a time-dependent background.

\section{WAVE ACTION}

Bretherton \& Garrett (1968) considered the propagation of linear waves in slowly varying, inhomogeneous, moving media in the WKB limit of short wavelengths and short wave periods. They took the waves to satisfy a dispersion relation of the form

$$
\omega=\Omega(\boldsymbol{k}, \lambda(\boldsymbol{r}, t)),
$$

where $\lambda(\boldsymbol{r}, t)$ is some slowly varying function of position and time. The group velocity of the waves is then

$$
c=\nabla_{k} \Omega,
$$

where $\nabla_{k}$ denotes the gradient operator in wavenumber space, and the frequency varies along a ray path according to the equation

$$
\frac{d \omega}{d t}=\frac{\partial \Omega}{\partial \lambda} \frac{\partial \lambda}{\partial t}
$$

where

$$
\frac{d}{d t}=\frac{\partial}{\partial t}+c \cdot \nabla
$$

Bretherton \& Garrett (1968) showed that for a wide class of conservative systems, including Alfvén waves in a time- 
dependent, inhomogeneous, moving medium,

$$
\frac{d}{d t}\left(\frac{\mathcal{E}_{\mathrm{w}}}{\omega^{\prime}}\right)+(\nabla \cdot c)\left(\frac{\mathcal{E}_{\mathrm{w}}}{\omega^{\prime}}\right)=0
$$

where $c$ is the group velocity,

$$
\omega^{\prime}=\omega-\boldsymbol{k} \cdot \boldsymbol{U}
$$

is the wave frequency measured in the local rest frame of the medium, $\boldsymbol{U}$ is the velocity of the medium, and $\mathcal{E}_{\mathrm{w}}$ is the energy density of the waves.

For the case of WKB Alfvén waves propagating in a radial magnetic field, $\mathcal{E}_{\mathrm{w}}=\rho_{0}\left\langle\left(z^{ \pm}\right)^{2}\right\rangle / 4, \Omega=k_{r}\left(U \pm v_{\mathrm{A}}\right), \quad \lambda=$ $U \pm v_{\mathrm{A}}$, and $\omega^{\prime}= \pm k_{r} v_{\mathrm{A}}$. Upon multiplying Equation (43) by $\omega$ and making use of Equation (41), we obtain

$$
\frac{d}{d t}\left(\frac{\omega \mathcal{E}_{\mathrm{w}}}{\omega^{\prime}}\right)+(\nabla \cdot \boldsymbol{c})\left(\frac{\omega \mathcal{E}_{\mathrm{w}}}{\omega^{\prime}}\right)=\frac{\mathcal{E}_{\mathrm{w}}}{v_{\mathrm{A}}} \frac{\partial}{\partial t}\left(v_{\mathrm{A}} \pm U\right) .
$$

To see how Equation (45) is recovered as a limiting case in our analysis, we multiply Equation (28) by $\rho_{0}\left(U \pm v_{\mathrm{A}}\right) /\left(4 v_{\mathrm{A}}\right)$ and simplify the resulting expression using Equation (22) and the identities $\boldsymbol{B}_{0} \cdot \nabla\left(\rho_{0} U / B_{0}\right)=-\partial \rho_{0} / \partial t$ and $\nabla \cdot\left(\rho_{0} \boldsymbol{v}_{\mathrm{A}}\right)=$ $-\rho_{0} \nabla \cdot \boldsymbol{v}_{\mathrm{A}}=(1 / 2) \boldsymbol{v}_{\mathrm{A}} \cdot \nabla \rho_{0}$. After some algebra, we obtain

$$
\begin{gathered}
\frac{\partial}{\partial t}\left[\frac{\rho_{0}\left(U \pm v_{\mathrm{A}}\right)\left\langle\left(z^{ \pm}\right)^{2}\right\rangle}{4 v_{\mathrm{A}}}\right]+\nabla \cdot\left[\frac{\hat{\boldsymbol{b}} \rho_{0}\left(U \pm v_{\mathrm{A}}\right)^{2}\left\langle\left(z^{ \pm}\right)^{2}\right\rangle}{4 v_{\mathrm{A}}}\right] \\
=\frac{\rho_{0}\left\langle\left(z^{ \pm}\right)^{2}\right\rangle}{4 v_{\mathrm{A}}} \frac{\partial}{\partial t}\left(U \pm v_{\mathrm{A}}\right)+\frac{\rho_{0}\left\langle z^{+} \cdot z^{-}\right\rangle}{4} \\
\times\left[\left(\frac{v_{\mathrm{A}}^{2}-U^{2}}{v_{\mathrm{A}}}\right)\left(\sigma+\frac{\hat{\boldsymbol{b}} \cdot \nabla \rho_{0}}{2 \rho_{0}}\right)-\frac{\left(U \pm v_{\mathrm{A}}\right)}{2 \rho_{0} v_{\mathrm{A}}} \frac{\partial \rho_{0}}{\partial t}\right] .
\end{gathered}
$$

In the limit of short wavelengths and small wave amplitudes, $\left\langle z^{+} \cdot z^{-}\right\rangle \rightarrow 0$. In this limit, Equation (46) reduces to (45). When $\partial \rho_{0} / \partial t, \partial U / \partial t$, and $\partial v_{\mathrm{A}} / \partial t$ vanish, subtracting the "minus version" of Equation (46) from the "plus version" of Equation (46) reproduces Equation (38) with the right-hand side of Equation (38) replaced by zero.

\section{CONCLUSION}

Conservation laws play a fundamental role in the study of turbulence, because they are among the few analytic results that can be used to gain insight into the physics of turbulent systems. For example, energy conservation underpins the concept of an energy cascade, in which nonlinear interactions among fluctuations transfer fluctuation energy in a loss-free manner from large scales to small scales. This idea ultimately explains why the turbulent heating rate can be determined solely from the properties of the turbulence at large scales (i.e., the inertial range or the outer scale), regardless of the mechanisms that dissipate the energy at small scales. Conservation of magnetic helicity in MHD turbulence leads to the concept of an inverse cascade of magnetic helicity, which plays an important role in turbulent dynamos (Frisch et al. 1975; Pouquet et al. 1976). In this paper, we have shown that a third conservation law, that of cross helicity, applies to non-WKB AWs and reflection-driven AW turbulence in the solar wind. This result is in some ways surprising, because non-WKB reflection converts $z^{ \pm}$fluctuations into $z^{\mp}$ fluctuations, thereby altering the cross-helicity content of the fluctuations. The total cross helicity is nevertheless conserved because the fluctuations exert a force on the background solar wind, which alters the cross-helicity content of the background flow.

Our finding that cross helicity is conserved by non-WKB AWs and reflection-driven AW turbulence is important for a few reasons. First, it implies that cross helicity can be exchanged between the fluctuations and the background flow without loss. Second, in contrast to the equation of "non-WKB wave-action conservation" derived by Heinemann \& Olbert (1980), cross-helicity conservation holds even when the background flow varies in time. (Also, as discussed in Section 3 , the equations of cross-helicity and energy conservation can be combined to recover Heinemann \& Olbert (1980)'s conservation law when the background solar wind is timeindependent.) Third, the coupled equations for the fluctuations and background flow in Section 2 can be solved numerically to provide new insights into the heating and acceleration of the solar wind by reflection-driven AW turbulence, and crosshelicity conservation provides a valuable benchmarking tool for such simulations. There is a growing interest in numerical simulations of the solar wind that incorporate AW turbulence (see, e.g., Usmanov et al. 2011, 2014; van der Holst et al. 2014), in part because of the upcoming launch of Solar Probe Plus. This pioneering mission will shed new light on the mechanisms that heat and accelerate the solar wind by providing the first-ever in-situ measurements of the solar-wind acceleration region. By providing new insights into one such mechanism (reflection-driven AW turbulence) as well as a valuable benchmarking tool for certain types of numerical codes, our results may ultimately contribute to a deeper understanding of the solar wind's origin.

This work was supported in part by NASA grants NNX11AJ37G and NNX15AI80G and NSF grants AGS1258998, AGS-1331355, and PHY-1500041.

\section{APPENDIX A THE NARROW-MAGNETIC-FLUX-TUBE APPROXIMATION}

As discussed in Section 2.1, our results apply under either of two different assumptions about the geometry of the background magnetic field. The background field can be either exactly radial throughout a region spanning a solid angle of order unity as seen from the Sun, or the background field can be approximately radial within a narrow magnetic flux tube centered on a radial magnetic field line. In this appendix, we consider the case of a narrow magnetic flux tube. We work in spherical polar coordinates and take $\theta=0$ to correspond to the exactly radial background magnetic field line that coincides with the axis of the magnetic flux tube. We restrict our analysis to a region within which

$$
\theta \ll 1 .
$$

We assume that

$$
B_{0 \phi}=\frac{\partial B_{0 r}}{\partial \phi}=\frac{\partial B_{0 \theta}}{\partial \phi}=0
$$

and define

$$
H(r)=\left|\boldsymbol{B}_{0}(r, \theta=0)\right| .
$$


The condition that $\nabla \cdot \boldsymbol{B}=0$ implies that $\partial B_{\theta} / \partial \theta=0$ at $\theta=0$. We require that $\nabla^{2} \boldsymbol{B}_{0}$ be finite, which implies that $\partial B_{0 r} / \partial \theta$ vanishes at $\theta=0$, so that

$$
B_{0 r}(r, \theta)=H(r)\left[1+\mathcal{O}\left(\theta^{2}\right)\right] .
$$

The condition $\nabla \cdot \boldsymbol{B}_{0}=0$ then implies that

$$
B_{0 \theta}=-\frac{\theta}{2 r} \frac{d}{d r}\left(r^{2} H\right)\left[1+\mathcal{O}\left(\theta^{2}\right)\right] .
$$

We assume that

$$
\frac{r}{H} \frac{d H}{d r} \sim \mathcal{O}(1)
$$

Equations (48), (50) and (51) imply that

$$
\hat{\boldsymbol{b}}=\left[\hat{\boldsymbol{r}}-\hat{\boldsymbol{\theta}} \frac{\theta}{2 r H} \frac{d}{d r}\left(r^{2} H\right)\right]\left[1+\mathcal{O}\left(\theta^{2}\right)\right],
$$

where $\hat{\boldsymbol{b}} \equiv \boldsymbol{B}_{0} / B_{0}$. It follows from Equation (53) that

$$
\nabla \cdot \hat{\boldsymbol{b}}=\sigma+\mathcal{O}\left(\frac{\theta^{2}}{r}\right)
$$

where

$$
\sigma=-\frac{1}{H} \frac{d H}{d r} .
$$

It also follows that for any vector $\boldsymbol{a}$,

$$
\boldsymbol{a} \cdot \nabla \hat{\boldsymbol{b}}=\frac{\sigma}{2} \boldsymbol{a}_{\perp}+\mathcal{O}\left(\frac{\theta a_{\perp}}{r}\right)+\mathcal{O}\left(\frac{\theta a_{\|}}{r}\right),
$$

where $a_{\|}=\hat{\boldsymbol{b}} \cdot \boldsymbol{a}$ and $\boldsymbol{a}_{\perp}=\boldsymbol{a}-a_{\|} \hat{\boldsymbol{b}}$.

The quantities $\nabla \times \hat{\boldsymbol{b}}$ and $\hat{\boldsymbol{b}} \cdot \nabla \hat{\boldsymbol{b}}$ relate to twist and curvature of magnetic field lines and satisfy the relations

$$
\hat{\boldsymbol{b}} \cdot \nabla \hat{\boldsymbol{b}}=\nabla_{\perp} \psi+\mathcal{O}\left(\frac{\theta^{2}}{r}\right)
$$

and

$$
\nabla \times \hat{\boldsymbol{b}}=\hat{\boldsymbol{b}} \times(\hat{\boldsymbol{b}} \cdot \nabla \hat{\boldsymbol{b}})
$$

where

$$
\psi=\frac{\theta^{2} r}{8}\left[2 \frac{d}{d r}(\sigma r)+(\sigma r-2) \sigma\right] .
$$

Equation (58) is not a vector identity, but is exact because of Equation (48). It follows from Equations (52), (54), (57) and (58) that

$$
|\nabla \times \hat{\boldsymbol{b}}| \sim|\hat{\boldsymbol{b}} \cdot \nabla \hat{\boldsymbol{b}}| \sim \mathcal{O}\left(\frac{\theta}{r}\right) \ll|\nabla \cdot \hat{\boldsymbol{b}}| \sim \mathcal{O}\left(\frac{1}{r}\right) .
$$

At several points in Sections 2 and 3, we average equations over surfaces that are everywhere perpendicular to $\boldsymbol{B}_{0}$, as described in Section 2.2. To specify these surfaces mathematically in the case of a narrow magnetic flux tube in which $\boldsymbol{B}_{0}$ is not exactly radial, we introduce the vector potential $\boldsymbol{A}_{0}$ associated with the background magnetic field and define Clebsch coordinates (Euler potentials) $\alpha$ and $\beta$ that are related to $\boldsymbol{A}_{0}$ through the equation $\boldsymbol{A}_{0}=\alpha \nabla \beta$, which yields

$$
\boldsymbol{B}_{0}=\nabla \alpha \times \nabla \beta .
$$

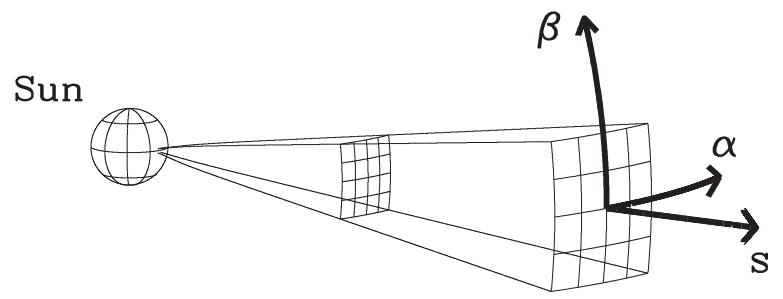

Figure 1. Clebsch coordinates $(\alpha, \beta, s)$. Surfaces of constant $s$ are perpendicular to $\boldsymbol{B}_{0}$. The values of $\alpha$ and $\beta$ are constant along field lines of $\boldsymbol{B}_{0}$.

Since $\boldsymbol{B}_{0} \cdot \nabla \alpha=\boldsymbol{B}_{0} \cdot \nabla \beta=0, \alpha$ and $\beta$ are constant along the magnetic field lines of $\boldsymbol{B}_{0}$. The particular Clebsch coordinates that we use are

$$
\alpha=[H(r)]^{1 / 2} x
$$

and

$$
\beta=[H(r)]^{1 / 2} y,
$$

where $(x, y, z)$ are Cartesian coordinates, and the positive $z$ axis coincides with $\theta=0$. When Equations (62) and (63) are substituted into Equation (61), the resulting value of $\boldsymbol{B}_{0}$ satisfies Equations (50) and (51), as required. We introduce a third coordinate $s$ such that surfaces of constant $s$ are perpendicular to $\boldsymbol{B}_{0}$, with $s=r$ at $\theta=0$ :

$$
s=r-\frac{\left(x^{2}+y^{2}\right)}{4 r^{2} H} \frac{d}{d r}\left[r^{2} H(r)\right]+\mathcal{O}\left(\theta^{4} r\right) .
$$

The $(\alpha, \beta, s)$ coordinate system is illustrated in Figure 1.

For any function $f(\alpha, \beta, s)$, we define $\langle f\rangle$ to be the average of $f$ over a surface of constant $s$ with $-\delta<\alpha<\delta$ and $-\delta<\beta<\delta$, where $\delta$ is a constant that fixes the width of the flux tube. Two such surfaces are sketched with cross-hatched lines in Figure 1. Thus,

$$
\langle f\rangle=\frac{1}{A} \int_{-\delta}^{\delta} d \alpha \int_{-\delta}^{\delta} d \beta \frac{f}{B_{0}},
$$

where

$$
A=\int_{-\delta}^{\delta} d \alpha \int_{-\delta}^{\delta} d \beta \frac{1}{B_{0}}
$$

is the area of the averaging surface.

Equations (50), (51), and (64) imply that $\left|\boldsymbol{B}_{0}\right|=$ $H(s)\left[1+\mathcal{O}\left(\theta^{2}\right)\right]$. We can thus rewrite Equation (65) as

$$
\langle f\rangle=\frac{1}{\Phi} \int_{-\delta}^{\delta} d \alpha \int_{-\delta}^{\delta} d \beta f\left[1+\mathcal{O}\left(\theta^{2}\right)\right],
$$

where

$$
\Phi=4 \delta^{2}
$$

is the magnetic flux through the averaging surface, which is independent of $s$. Equation (64) implies that $|\nabla s|=1+\mathcal{O}\left(\theta^{2}\right)$, so that

$$
\hat{\boldsymbol{b}} \cdot \nabla f=\frac{\partial f}{\partial s}\left[1+\mathcal{O}\left(\theta^{2}\right)\right]
$$


It follows from Equation (67) that

$$
\left\langle\frac{\partial f}{\partial s}\right\rangle=\frac{\partial}{\partial s}\langle f\rangle\left[1+\mathcal{O}\left(\theta_{0}^{2}\right)\right],
$$

where $\theta_{0}$ is the value of $\theta$ at the middle of one of the edges of the averaging surface-e.g., at coordinates $(\alpha, \beta, s)=(\delta, 0, s)$.

We say that a scalar function $g$ satisfies periodic boundary conditions in the plane perpendicular to $\boldsymbol{B}_{0}$ if it obeys the relations

$$
g(\delta, \beta, s)=g(-\delta, \beta, s) \quad g(\alpha, \delta, s)=g(\alpha,-\delta, s)
$$

for all $\alpha \in(-\delta, \delta)$, all $\beta \in(-\delta, \delta)$, and all $s$. We say that a vector $\boldsymbol{a}$ satisfies periodic boundary conditions in the plane perpendicular to $\boldsymbol{B}_{0}$ if the vector's components $\boldsymbol{a} \cdot \nabla \alpha, \boldsymbol{a} \cdot \nabla \beta$, and $\boldsymbol{a} \cdot \nabla s$ satisfy the periodicity relations in Equations (71). If a vector $\boldsymbol{a}_{\perp}$ is periodic in the plane perpendicular to $\boldsymbol{B}_{0}$ and satisfies $\boldsymbol{a}_{\perp} \cdot \boldsymbol{B}_{0}=0$ everywhere, then Stokes' theorem can be used to show that

$$
\left\langle\nabla \cdot \boldsymbol{a}_{\perp}\right\rangle \sim \frac{\theta_{0}\left\langle\left|\boldsymbol{a}_{\perp}\right|^{2}\right\rangle^{1 / 2}}{r},
$$

where we have assumed that the characteristic length scale of $\boldsymbol{a}_{\perp}$ perpendicular to $\boldsymbol{B}_{0}$ is $\sim \theta_{0} r$. The rms value of $\nabla \cdot \boldsymbol{a}_{\perp}$ on the averaging surface is $\sim\left\langle\left|\boldsymbol{a}_{\perp}\right|^{2}\right\rangle^{1 / 2} /\left(\theta_{0} r\right)$. Equation (72) thus implies that the average of $\nabla \cdot \boldsymbol{a}_{\perp}$ is reduced relative to its $\mathrm{rms}$ value by a factor of $\sim \theta_{0}^{2}$. (This reduction factor would be even smaller if the characteristic length scale of $\boldsymbol{a}_{\perp}$ perpendicular to $\boldsymbol{B}_{0}$ were much smaller than $\theta_{0} r$.) This reduction enables us to drop averaged quantities of the form $\left\langle\nabla \cdot \boldsymbol{a}_{\perp}\right\rangle$ in Section 2, because they contribute only higher-order corrections to the equations presented.

\section{APPENDIX B \\ ELSASSER STREAM FUNCTIONS AND VORTICITIES}

We define the Elsasser stream functions $\zeta^{ \pm}$and the Elsasser vorticities $\Omega^{ \pm}$through the equations

$$
z^{ \pm}=\hat{\boldsymbol{b}} \times \nabla \zeta^{ \pm} \quad \Omega^{ \pm}=\nabla_{\perp}^{2} \zeta^{ \pm},
$$

where

$$
\nabla_{\perp}^{2} f \equiv \nabla \cdot\left(\nabla_{\perp} f\right)=\nabla \cdot[\nabla f-\hat{\boldsymbol{b}}(\hat{\boldsymbol{b}} \cdot \nabla f)]
$$

for any function $f$. By taking the cross product of Equation (26) with $\hat{\boldsymbol{b}}$ and then taking the divergence of the resulting equation, we can rewrite Equation (26) in the form

$$
\begin{gathered}
\frac{\partial}{\partial t} \Omega^{ \pm}+\left(U \pm v_{\mathrm{A}}\right)\left(\hat{\boldsymbol{b}} \cdot \nabla \Omega^{ \pm}+\frac{\sigma}{2} \Omega^{ \pm}\right) \\
=\frac{\sigma}{2}\left(-U \pm v_{\mathrm{A}}\right) \Omega^{\mp} \\
+\frac{1}{2}\left(\nabla \cdot v_{\mathrm{A}} \mp \frac{1}{2} \nabla \cdot \boldsymbol{U}\right)\left(\Omega^{+}-\Omega^{-}\right) \\
-\frac{1}{2}\left(\left\{\zeta^{-}, \nabla_{\perp}^{2} \zeta^{+}\right\}+\left\{\zeta^{+}, \nabla_{\perp}^{2} \zeta^{-}\right\} \pm \nabla_{\perp}^{2}\left\{\zeta^{-}, \zeta^{+}\right\}\right),
\end{gathered}
$$

where

$$
\{f, g\} \equiv \hat{\boldsymbol{b}} \cdot\left(\nabla_{\perp} f \times \nabla_{\perp} g\right) .
$$

Here, we have assumed that either $\theta \ll 1$ for the case in which the background magnetic field corresponds to a narrow magnetic flux tube or $L_{\mathrm{c} \perp} \ll r$ for the case in which $\boldsymbol{B}_{0}$ is radial throughout a region spanning a solid angle of order unity, where $L_{\mathrm{c} \perp}$ is the correlation length of the fluctuations perpendicular to $\boldsymbol{B}_{0}$. Equation (75) generalizes Equation (A4) of van Ballegooijen et al. (2011) to account for the background flow $\boldsymbol{U}$. Equation (75) is the same equation that was solved numerically by Perez \& Chandran (2013). (Note that the minus sign on the right-hand side of their Equation (10), which was erroneous, was a typo in their paper, not an error in their code.) The form of the nonlinear term on the last line of Equation (75) is the same as in the RMHD equations derived by Schekochihin et al. (2009), except that our $\zeta^{ \pm}$corresponds to their $\zeta^{\mp}$. In the homogeneous-background limit (in which $\sigma, \nabla \cdot \boldsymbol{v}_{\mathrm{A}}$, and $\nabla \cdot \boldsymbol{U}$ vanish), Equation (75) reduces to Equation (21) of Schekochihin et al. (2009).

\section{REFERENCES}

Belcher, J. W., \& Davis, L., Jr. 1971, JGR, 76, 3534

Bretherton, F. P., \& Garrett, C. J. R. 1968, RSPSA, 302, 529

Bruno, R., \& Carbone, V. 2005, LRSP, 2, 4

Chandran, B. D. G. 2008, PhRvL, 101, 235004

Chandran, B. D. G., \& Hollweg, J. V. 2009, ApJ, 707, 1659

Chandran, B. D. G., Quataert, E., Howes, G. G., Xia, Q., \& Pongkitiwanichakul, P. 2009, ApJ, 707, 1668

Coleman, P. J. 1968, ApJ, 153, 371

Coles, W. A., \& Harmon, J. K. 1989, ApJ, 337, 1023

Cranmer, S. R., \& van Ballegooijen, A. A. 2005, ApJS, 156, 265

De Pontieu, B., McIntosh, S. W., Carlsson, M., et al. 2007, Sci, 318, 1574

Dmitruk, P., Matthaeus, W. H., Milano, L. J., et al. 2002, ApJ, 575, 571

Frisch, U., Pouquet, A., Leorat, J., \& Mazure, A. 1975, JFM, 68, 769

Goldstein, M. L., Roberts, D. A., \& Matthaeus, W. H. 1995, ARA\&A, 33, 283

Heinemann, M., \& Olbert, S. 1980, JGR, 85, 1311

Hollweg, J. V., Cranmer, S. R., \& Chandran, B. D. G. 2010, ApJ, 722, 1495

Hollweg, J. V., \& Isenberg, P. A. 2007, JGRA, 112, 8102

Iroshnikov, P. S. 1963, AZh, 40, 742

Kraichnan, R. H. 1965, PhFl, 8, 1385

Markovskii, S. A., \& Hollweg, J. V. 2002, JGRA, 107, 21

Matthaeus, W. H., Zank, G. P., Oughton, S., Mullan, D. J., \& Dmitruk, P. 1999, ApJL, 523, L93

Parker, E. N. 1965, SSRv, 4, 666

Perez, J. C., \& Chandran, B. D. G. 2013, ApJ, 776, 124

Pouquet, A., Frisch, U., \& Leorat, J. 1976, JFM, 77, 321

Schekochihin, A. A., Cowley, S. C., Dorland, W., et al. 2009, ApJS, 182, 310

Tu, C., \& Marsch, E. 1995, SSRv, 73, 1

Usmanov, A. V., Goldstein, M. L., \& Matthaeus, W. H. 2014, ApJ, 788, 43

Usmanov, A. V., Matthaeus, W. H., Breech, B. A., \& Goldstein, M. L. 2011, ApJ, 727, 84

van Ballegooijen, A. A., Asgari-Targhi, M., Cranmer, S. R., \& DeLuca, E. E. 2011, ApJ, 736, 3

van der Holst, B., Sokolov, I. V., Meng, X., et al. 2014, ApJ, 782, 81

Velli, M. 1993, A\&A, 270, 304

Velli, M., Grappin, R., \& Mangeney, A. 1989, PhRvL, 63, 1807

Verdini, A., Grappin, R., Pinto, R., \& Velli, M. 2012, ApJL, 750, L33

Verdini, A., \& Velli, M. 2007, ApJ, 662, 669

Zank, G. P., Dosch, A., Hunana, P., et al. 2012, ApJ, 745, 35

Zhou, Y., \& Matthaeus, W. H. 1989, GeoRL, 16, 755

Zhou, Y., \& Matthaeus, W. H. 1990, JGR, 95, 14881 